\title{
Molecular motion on ice
}

\author{
With advanced approaches to sample preparation and data analysis, scientists doing cryo-EM are starting to infer \\ molecular movements from samples that are, literally, frozen.
}

\section{Amber Dance}

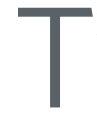
he RNA polymerase that transcribes DNA is about as central to the central dogma of molecular biology as you can get. And yet, after decades of study, there remain mysteries, says Ruth Saecker, a biophysical chemist at the Rockefeller University in New York.

"There was still this huge, quoteunquote, 'open question' of how the RNA polymerase machinery opens DNA," she says. It's something that happens so fast, structural biologists couldn't get a good picture of the event.

Now, thanks to the latest innovations in cryo-electron microscopy (EM) sample preparation, she and her colleagues in Seth Darst's laboratory are close to an answer. With collaborators at the New York Structural Biology Center, they used a device called Spotiton to paint a cryo-EM grid with separate spurts of the polymerase and target DNA as the grid was plunging toward liquid ethane to freeze the molecules in place. The polymerase and DNA had about $150 \mathrm{~ms}$ to mix and begin to interact, allowing Saecker to see the enzyme just as it recognized a promoter.

"It's a breakthrough in my field," she says. "There's no question it'll be a breakthrough for many other biological processes."

The field of cryo-EM, which allows researchers to observe single particles and sum the data to produce three-dimensional molecular structures, has long focused on getting a structure, singular. But biomolecules may enter transient states, such as Saecker's opening of DNA by polymerase, or move around to do their jobs. Viruses shed their capsids. Actomyosin contracts to move muscles. Ion channels open and close. Therefore, one structure is far from the whole story, says Georgios Skiniotis, a structural biologist at Stanford University in California. That story requires the entire ensemble of structures a protein can adopt.

"You need the dynamics, you need to show the system in different states and how moving parts go left and right, in an effort to understand how it works," Skiniotis says.

To comprehend proteins in action, structural biologists must access rare or transient conformations, such as those that

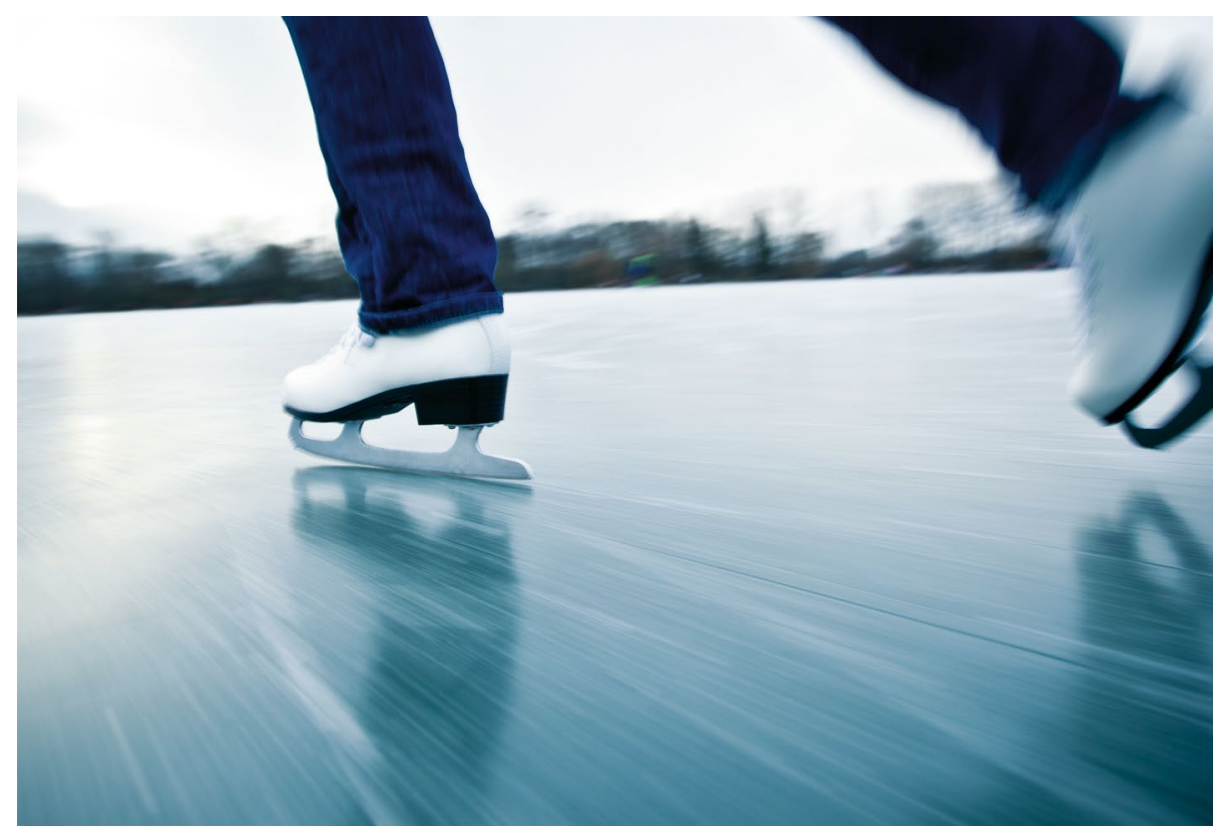

Though cryo-EM images are frozen in time, with the right techniques, they can offer clues to the motion of biomolecules. Credit: ViktorCap / iStock / Getty Images Plus

occur at the start of a reaction and rapidly disappear. They can do so, in great part, thanks to recent advances in cryo-EM, which was named Nature Method's Method of the Year 2015. With sprayers such as Spotiton, researchers can capture biomolecules in a thin layer of liquid - tens to hundreds of nanometers - on a sample grid before quickly freezing it. Electron microscopes have improved dramatically, with direct electron detectors that improve resolution and automation software that allows scientists to image hundreds of thousands of particles with relative ease. And modern software makes it possible to parse the particles into multiple substructures.

"Cryo-EM has recently achieved star status in actually getting very high resolution," says Crina Nimigean, a biophysicist at Weill Cornell Medicine in New York. Scientists can now routinely obtain structures at better than $2.5 \AA$ resolution.

To apply these new and improved tools to rare conformers, scientists typically take one of two tacks. Some assess the reactions they're interested in at equilibrium. While most biomolecules will have reached the end of the reaction, some rare intermediates still exist. By imaging enough particles and using the latest software, researchers can identify and build structures for those intermediates, each contributing a snapshot of the reaction. "You just take a big old mixed pot," says Rebecca Thompson, facility manager for cryo-EM at the University of Leeds in the UK, and "sort it all out."

Classic algorithms offer up structures of discrete steps in a reaction, but newer software models protein subunits that wave or spin as continuous motion, like a movie. "There's a lot of information to be gleaned just from looking at the equilibrium distribution," says John Rubinstein, a structural biologist at The Hospital for Sick Children in Toronto.

Others prefer to stack the deck toward early intermediates with time-resolved techniques, freezing the sample just milliseconds into the reaction time before sorting the ensemble with the same 


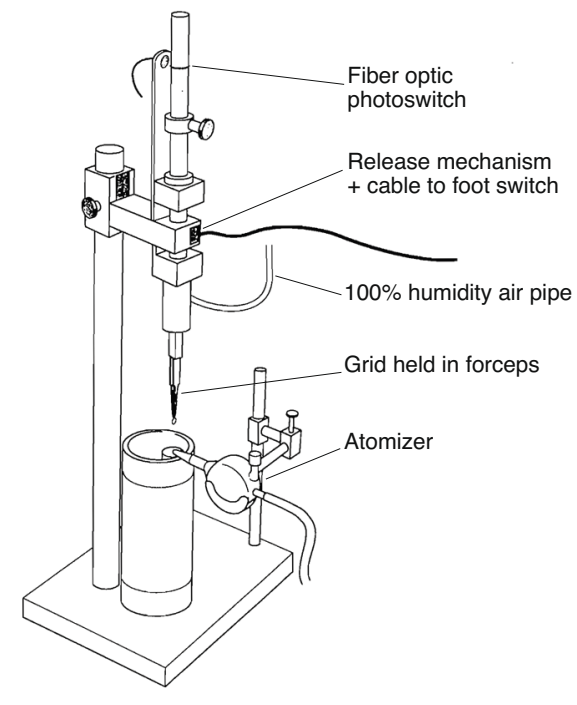

In an early attempt to freeze EM grids shortly after mixing, researchers designed this apparatus to spray the grid with a reactant right before it hits the cryogenic liquid. Many modern devices work similarly. Adapted with permission from ref. ${ }^{5}$, Elsevier.

algorithms. Devices to do this are just beginning to be commercialized, and some research groups are publishing instructions to build them on the cheap, so this technique is poised to reach widespread use in upcoming years.

For millisecond-length reactions, "it already seems like it's open season, if you have the right technology and the right systems," says James Fraser, a structural biologist at the University of California, San Francisco. "The dream is to be able to make sort of molecular movies of proteins as they do their functions."

Certainly, other techniques can do similar tasks: mass spectrometry, NMR spectroscopy, and FRET microscopy give the kinetics of a reaction, and X-ray crystallography offers dynamics. Of those, only X-ray studies build an image of molecules, as cryo-EM does. But the proteins are constrained by the crystal lattice, unable to make large movements. And an X-ray structure is an average of all particles, so rare intermediates are invisible. Cryo-EM can now showcase the glorious heterogeneity of biomolecular structures.

"It's really the next frontier of structural biology," says Rubinstein. "How does that structure evolve over time - that's where life happens. That's biology."

\section{Sorting structures}

For Eva Nogales, X-ray crystallography has never been an option. The biophysicist at the University of California, Berkeley, studies the human transcription factor IID, which recognizes a promoter to initiate RNA synthesis. It's a 20-part protein complex one megadalton in weight; crystallizing that monster was a nonstarter. In a recent study, Nogales used cryo-EM at equilibrium alongside other techniques to sort out the complex's diverse conformations including the $150-\AA$ swing of one section ${ }^{1}$.

A popular sorting technique ${ }^{2}$ was developed by Sjors Scheres, a structural biologist at the Medical Research Council (MRC) Laboratory of Molecular Biology in Cambridge, UK. It's built into his open-source RELION software, and many other packages as well.

First, the software lumps all the particles together, obtaining an overall structure. Any spots where the protein can move will show up blurred.

Then, the software randomly divides the dataset into several groups: say, A, B, C and D. For each individual particle, the software asks, is it a better match for group A, or B or $\mathrm{C}$ or $\mathrm{D}$ ? If there's a better match in another group, as based on a statistical method called maximum likelihood estimation, the program will move that particle to the new group. After about 25 rounds, this sorts the proteins into groups with common structures.

The program relies on the human user to decide how many groups to start with. Too few will lead to mixed-up results, but too many is OK, says Scheres. If there are more groups than different structures, then two groups might represent the same structure, or an extra group might just be nonsense.

Nogales took this approach with TFIID, but the complex was so large that one sort was not sufficient. The researchers had to use their own knowledge and intuition to manage multiple rounds of subgroupings - first two sets, then more, and finally the positions of the complex's three lobes. This analysis revealed that the complex moves through at least five structural states.

While the team had a good idea of what to expect, some results were surprising, Nogales says. For example, two of the TFIID lobes have very similar structures, but only one lobe moved while the other stayed put.

\section{Bodies in motion}

This maximum likelihood approach works well, Scheres says, when a protein adopts discrete states. But many biomolecules move continually, rotating or morphing all the time. So the new front line in algorithm development, he says, is to model that constant motion.
Scheres took his first stab at it with a technique he calls "multi-body refinement." In this case, researchers divide their biomolecule or complex of interest up into parts. Each part, it's assumed, is rigid, unmovable, while the joints between these parts can rotate or swing. Scheres tested the approach on data from a ribosome, splitting it into three segments and achieving structures for elements that had never been interpretable before.

Others have adopted the technique, but Scheres admits he took a shortcut by assuming rigidity in a protein's parts, so the method misses plenty of wiggle. And if a user defines the rigid parts poorly, the program doesn't produce useful results. "Ultimately, this is not the right solution yet," he says. He's working on improvements.

Structural biologists are also excited about a new algorithm in a program called CryoSPARC, free to academics from Structura Biotechnology of Toronto. Instead of dividing proteins into discrete conformations or parts, it can analyze continuous motion. Company CEO Ali Punjani, a graduate student at the University of Toronto, says he used the simplest possible model, which he calls "3D variability analysis." ${ }^{4}$ For a given protein, the algorithm assigns an 'average' structure, then represents every other possible conformation as a set of gradual deviations along a continuum.

Even that 'simple' solution took two years to get right. "This is definitely not the first word or the last word in this area," Punjani says. "It's a step forward." While his current algorithm is certainly useful, he says he's already working on more complex models.

Machine learning is also beginning to make inroads in cryo-EM analysis. The advantage of machine learning is that the data train the algorithm, so human bias is reduced, says Abbas Ourmazd, a physicist at the University of Wisconsin-Milwaukee. But if the data used for training differ in some important way from the data being analyzed, the program may make mistakes.

No matter the analysis technique, humans always interpret the final structures. It's appealing to build pathways, even movies, from one structure to the next, but that path is fraught with danger, says Ourmazd. Does conformation C always come after conformation $\mathrm{B}$ in the reaction, for example, or might it sometimes run straight to D? "Who knows what happens in between?" says Ourmazd.

Ultimately, cryo-EM tells researchers which structures are possible - but doesn't confirm which are biologically relevant. Thus, experts caution cryo-EM users not to go too far in their interpretations. 


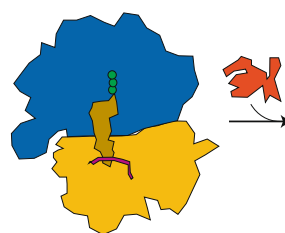

Termination complex $t=0 \mathrm{~ms}$

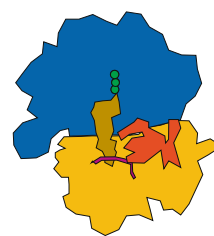

Pre-accommodated RF-ribosome complex $t \approx 20 \mathrm{~ms}$

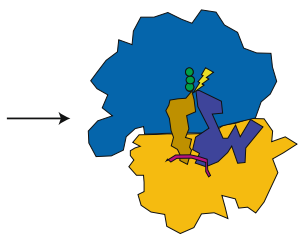

Accommodated RF-ribosome complex $t \approx 60 \mathrm{~ms}$

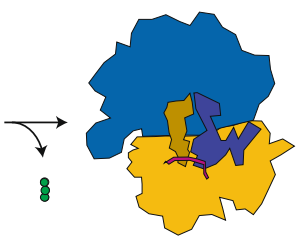

Peptide released RF-ribosome complex later time point
Researchers used time-resolved cryo-EM to build a model for the ribosome's release of a completed peptide when a stop codon enters the active site. A release factor (RF), in compact form (red), binds within milliseconds. That factor then extends (purple), releasing the peptide (green). Adapted from ref. under a Creative Commons Attribution 4.0 International license.

\section{The need for speed}

Similar niggling worries have long plagued Joachim Frank, a physicist at Columbia University in New York. He's been studying the structure of the ribosome for decades, and wants to understand what goes down during quick processes, such as the activation of translation termination when a stop codon arrives. Using tricks such as GTP analogs, he's managed to lock the ribosome and its interactors in various intermediate states for structural evaluation. be artificial, so he has to wonder whether he's deduced the real conformation.

What he'd like to do is freeze the sample shortly after a biological process begins, so he can see the native intermediates that appear in the first second of a reaction. But preparing a sample takes several seconds. That's where new, time-resolved techniques come in.

The idea is not a new one. "A lot of proteins flicker into the active state and back out," says Bridget Carragher, a structural biologist at the New York Structural Biology Center. "People have always wanted to know how proteins move, and what they do, and catch them in these short-lived states."

Back in the 1990s, Nigel Unwin and John Berriman at the MRC spread acetylcholine receptors on EM grids, then mounted these on a dropping device, with liquid ethane at the bottom to chill the sample. As the grids plunged, the device sprayed them with acetylcholine, giving the receptors milliseconds to respond before freezing ${ }^{5}$.

"That was pretty groundbreaking," says University of Leeds, "but not many people have gone on to do much else ... mainly because it's hard to do."

Sample preparation, even without cryo-EM. The liquid layer must be just a smidge thicker than the biomolecule itself. But those locked states have the potential to Stephen Muench, a structural biologist at the mixing, remains a huge challenge in
When proteins or other biomolecules hit the surface where liquid meets air, they may unfold and aggregate. Many grids end up tossed in the trash, useless.

Today, many scientists use the Vitrobot. Researchers pipette a few microliters of sample onto the grid, which is automatically blotted to create a thin liquid film and quickly plunged in liquid ethane. Peter Peters, a nanobiologist at Maastricht University in the Netherlands, and his colleagues built a new sample prep device. Called the VitroJet, it's marketed by CryoSol-World of Maastricht. It uses a tiny pin to pick up less than a nanoliter of sample, then scribes it in a neat line or oval on a grid, before freezing by jets of liquid ethane.

With the recent improvements in sample preparation and microscopes, researchers can collect many more protein images, faster, than when those MRC teams were working. That's made time-resolved sample prep worth a revisit, says Muench.

For example, Peters envisions a VitroJet that draws a cross, with one reactant in the vertical line and one in the horizontal, so they would mix at the point where they meet before freezing. Many other scientists are also pushing the limits of mixing within milliseconds of freezing.

Researchers at the New York State Department of Health's Wadsworth Center in Albany, New York, developed a time-resolved method they call "mix and spray," which Frank's group at Columbia has improved. Combining reactants before they hit the grid allows the team to control the mixing process, says Frank. His technique runs the reactants through a microfluidic chip to blend them. The researchers can manage how long the components mix by the length of the microfluidic channel; a longer path will give more time. Upon exiting the channel, the biomolecules are sprayed onto a grid as it plunges toward a pool of liquid ethane. Frank says he can capture reactions within $15 \mathrm{~ms}$ to $1 \mathrm{~s}$ after mixing, and he hopes to achieve $0.5 \mathrm{~ms}$ or faster.

He recently used this approach to study the release factors that free a completed peptide from the ribosome ${ }^{6}$. These release factors normally adopt a scrunched shape, but to catalyze peptide release, they must reach two spots $70 \AA$ apart. Using time-resolved cryo-EM, Frank's team observed release factors bound to the ribosome, but still scrunched. They found that after $24 \mathrm{~ms}$, one-quarter of release factors remained in the compact, unstretched state, while the rest had stretched. By $60 \mathrm{~ms}$, they'd all extended.

In July, a team of collaborators from Europe and South Korea reported a similar setup, mixing two components in a series of switchbacks in a microfluidic chip before spraying a fine mist into an even layer on a cryo-EM grid ${ }^{7}$. Their machine can freeze samples within tens to hundreds of milliseconds of mixing.

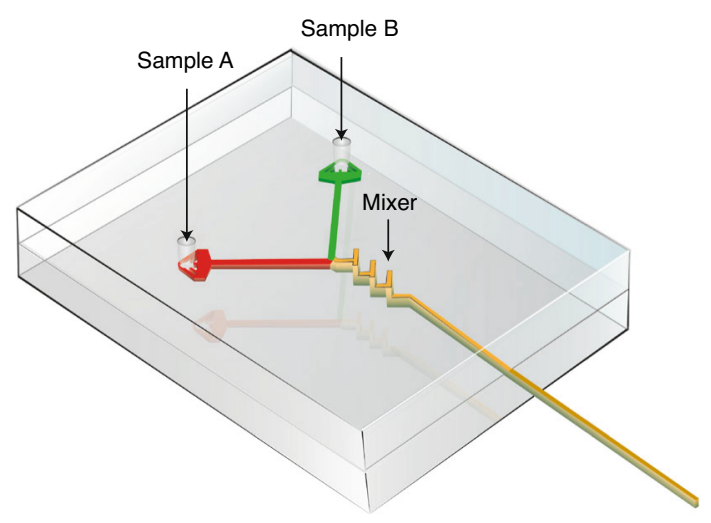

By running two components along a series of switchbacks, this microfluidic chip mixes reactants before their deposition on an EM grid. Adapted from ref. ${ }^{7}$ under a Creative Commons Attribution 4.0 International license. 


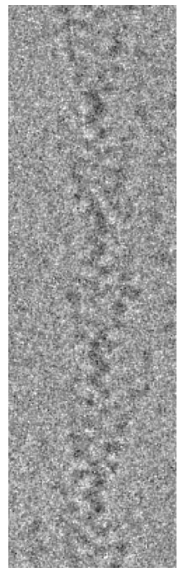

Actomyosin

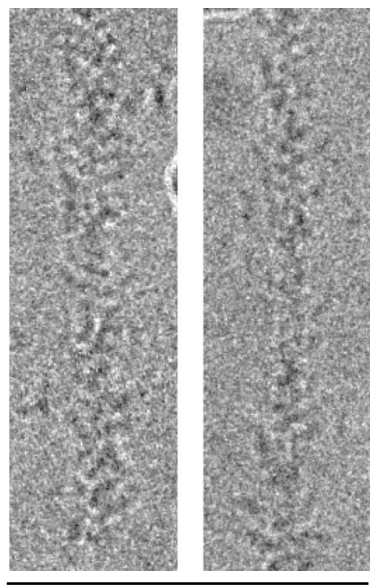

Actomyosin + ATP $(10 \mathrm{~ms})$

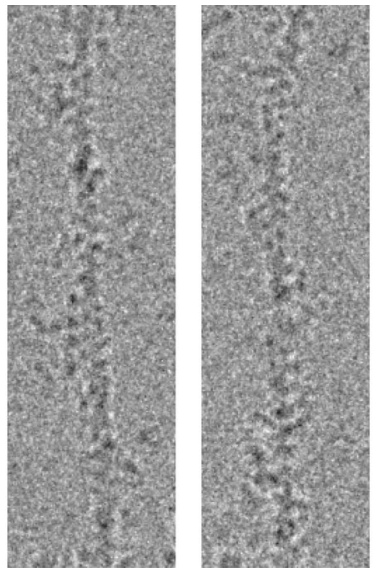

Actomyosin + ATP $(300 \mathrm{~ms})$

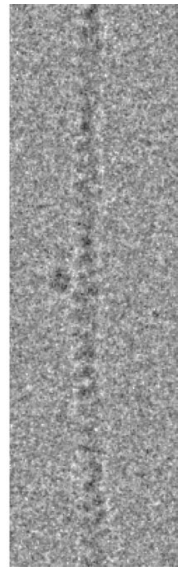

Actin

Upon addition of ATP, myosin dissociates from actomyosin fibers in less than a second. Using time-resolved cryo-EM, a team at the University of Leeds observed this happening. A pure actin filament (right) is shown for comparison. Credit: David Klebl, University of Leeds, Leeds, UK

\section{Spot it on}

A downside of the microchip mixing approach is that it requires a lot of sample; some is left in the microfluidic channel or sprayed outside the grid, says Frank. It's relatively straightforward to purify plenty of ribosome and associated RNA, but other biomolecules are dearer.

Frank says he's working on ways to minimize the protein needed for his device. Carragher prefers to spread the sample evenly first and mix on the grid, using up less protein in the process.

It started with a device for quick freezing of cryo-EM specimens, without mixing: Spotiton, developed by Carragher and Clint Potter, with whom she directs the center's EM lab, and their team. As EM grids rush toward a liquid ethane bath, the Spotiton device uses an inkjet printer nozzle to deposit fine droplets, on the order of 50 picoliters each, in a streak. Their special trick is to use homemade grids bristling with nanowires to wick the liquid like a paper towel. This creates "a nice even stripe of ice," Carragher says. The grids vary and the wicking doesn't always work perfectly, but when it does, the entire sample should be available for imaging.

By adding a second nozzle to Spotiton, Carragher's group managed to deposit two components, which interact via Brownian motion on the grid for 90-150 ms before freezing. This is the device that produced Saecker's RNA polymerase data. Carragher's team and collaborators have also imaged transient states of an ion channel, dynamin, and the ribosome (ref. ${ }^{8}$, this issue).

So far, Saecker has just obtained enough images to prove she will be able to see RNA polymerase as it opens DNA. Imaging more biomolecules should reveal the process more clearly.

"We're really excited to continue it," says Saecker. "We have so much to learn."

Researchers don't go into these time courses blindly; ideally, they already know plenty about the processes they're after. Kinetics - from spectroscopy, for example - will tell scientists how long to mix before freezing. Saecker notes that the NYSBC's Spotiton is set up at room temperature. And to get enough particles on the grid, she needed reactant concentrations of 2-8 mg per milliliter. So she had to ensure her buffers and biomolecules would behave under those conditions.

\section{Build your own}

Carragher's first-generation Spotiton, with just one nozzle, is already available commercially as the chameleon from SPT Labtech in Melbourne, UK. And Frank is working with Hummingbird Scientific in Lacey, Washington, to bring his device to market.

Other scientists prefer the do-it-yourself approach. "You don't need any specialized equipment," says Rubinstein.

He studies rotary ATPases, for which motion is a key element. "They literally spin as part of their catalytic mechanism," he says.

He's working on a time-resolved cryo-EM device built from 3D-printed materials, consumer-grade electronics and a single-board Raspberry Pi computer, all for about US\$750. Instructions and plans for the single-component system are available on Github. He also uses a wicking mechanism, with a glass fiber filter on the back of conventional grids to create a broad, even liquid layer. As a play on Spotiton, he named his initial, single-sample device "Back-it-up." He's able to freeze samples within tens of milliseconds of their application.

In Leeds, one of Thompson's favorite parts of her lab is the corner scattered with pond misters, tubing and other hardware, where they're building a time-resolved cryo-EM device, or TED. ${ }^{10}$ "It looks like in the best possible way - somebody's shed," she says.

The team borrowed spray tech from X-ray crystallography - where it's possible to image reactions within femtoseconds to make it reliably fast, says Muench, who's leading the project along with Howard White of Eastern Virginia Medical School in Norfolk. Using a nozzle with one component coming down a central channel and another coming from the sides, they can mix just as droplets are formed. And the TED is fast: the team has gone as low as $6 \mathrm{~ms}$ from mixing to freezing ${ }^{11}$, and Thompson thinks they can reach a single millisecond.

Graduate student David Klebl used the TED to analyze actomyosin after he added ATP, causing the myosin part of the complex to dissociate and leaving a naked actin fibril. After $10 \mathrm{~ms}$, most of the myosin was still attached to actin, but the majority of it was gone by $300 \mathrm{~ms}$.

Which device to choose? It depends on one's budget to buy or technical skills to build, but also on the time course at hand. To squeeze the most data out of precious samples, something like Spotiton's even application could be the right ticket. But if speed is a priority, the TED might be a better selection. "Our device isn't going to win any competition in terms of the prettiness of the grids," Thompson admits, "but it's going to make grids very quickly."

\section{The whole story}

Radostin Danev, a physicist at the University of Tokyo, has not tried time-resolved sample prep yet, but he's excited about the possibilities that both this approach and the latest analysis software offer. "I'm sure that it will be one, definitely, big part of the field," he says. Using the computational methods alone is particularly appealing, he adds, as only one prep is required to find a variety of protein states.

These techniques will not only reveal the machinery behind basic biological concepts, but also could be used to develop medications, says Skiniotis. For example, one might determine how rigid a structure is when bound to different candidate 
drugs. Plus, the short-lived intermediates themselves might be useful drug targets.

Researchers can also combine cryo-EM with other techniques, says Muench. While scientists have often divided themselves into camps - the EM experts, the X-ray folks and so on - merging techniques will give researchers the most complete picture of molecular movements. Spectroscopy, crystallography and atomic force microscopy could all add to what cryo-EM can provide, he says. "That's the future for me," he says. "It's just blending of the techniques."

There's room for molecular dynamics simulations too, notes Skiniotis: such simulations might inform cryo-EM analyses, and vice versa.

Meanwhile, the methods to prepare cryo-EM samples quickly and to analyze the ensemble on a grid have plenty of room to grow. "We're still in our baby steps," says Skiniotis. But with ongoing improvements, and a multitude of approaches, scientists can finally start to build the whole story of a protein's living, moving cycle - even on ice.

\section{Amber Dance ${ }^{凶}$}

Los Angeles, CA, USA.

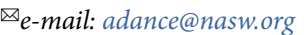

Published online: 19 August 2020 https://doi.org/10.1038/s41592-020-0940-7

References

1. Patel, A. B. et al. Science 362, eaau8872 (2018).

2. Scheres, S. H. W. et al. Structure 15, 1167-1177 (2007).

3. Nakane, T., Kimanius, D., Lindahl, E. \& Scheres, S. H. Elife 7, e36861 (2018).

4. Punjani, A. \& Fleet, D. J. Preprint at bioRxiv https://doi.org/ 10.1101/2020.04.08.032466 (2020).

5. Berriman, J. \& Unwin, N. Ultramicroscopy 56, 241-252 (1994).

6. Fu, Z. et al. Nat. Commun. 10, 2579 (2019).

7. Mäeots, M.-E. et al. Nat. Commun. 11, 3465 (2020).

8. Dandey, V. P. et al. Nat. Methods https://doi.org/10.1038/s41592020-0925-6 (2020).

9. Tan, Y. Z. \& Rubinstein, J. L. Preprint at bioRxiv https://doi.org/ 10.1101/2020.05.03.075366 (2020).

10. Kontziampasis, D. et al. IUCrJ 6, 1024-1031 (2019).

11. Klebl, D. P. et al. Preprint at bioRxiv https://doi.org/10.1101/ 2020.05.14.095372 (2020) 